\title{
FUNCTIONS AUTOMORPHIC ON LARGE DOMAINS
}

\author{
BY
}

\author{
DAVID A. JAMES
}

\begin{abstract}
For a discontinuous group $\Gamma \subset \mathrm{SL}(2, R)$, Poincaré produced a corresponding nonconstant automorphic form, meromorphic on the open upper half plane $\Pi^{+}$. When the domain of meromorphicity grows larger than $\mathbf{n}^{+}$, the type of group which can support an automorphic form is restricted, and the corresponding forms are generally quite simple. A complete analysis of this phenomenon is presented, with examples which show results are best possible.
\end{abstract}

I. Introduction. Let $\Gamma$ be a group under multiplication of two by two matrices with real entries and determinant one. To each matrix $M=\left(\begin{array}{ll}a & b \\ c & d\end{array}\right)$ there is a corresponding linear fractional transformation $M z=(a z+b) /(c z+d)$. A function $f(z)$ is an automorphic form of degree $r$ with respect to $\Gamma$ on some $\Gamma$-invariant set $\Omega$ if $f$ is meromorphic on $\Omega$ and

$$
f(z)=(c z+d)^{r} f(M z), \quad \forall M=\left(\begin{array}{ll}
a & b \\
c & d
\end{array}\right) \in \Gamma \text { and } \forall z \in \Omega .
$$

If $r=0, f(z)$ is an automorpbic function. If $v$ is a function acting on the elements of $\Gamma$ and $f(z) v(M)=(c z+d)^{r} f(M z), \forall M=\left(\begin{array}{ll}a & b \\ c & d\end{array}\right) \in \Gamma$ and $\forall z \in \Omega$, then $v$ is the multiplier system associated with the automorphic form $f$. Normally an automorphic form is required to have a certain growth condition within angles at parabolic vertices [5, pp. 76, 77], but this condition shall be deleted since it plays no role in the material presented here. For $M \neq \pm I$, the equation $M z=z$ has two solutions (which may coincide) in the extended complex plane. If these two fixed points $\zeta_{1}$ and $\zeta_{2}$ of $M$ are distinct and finite, $K$ is defined for each $M$ by $\left(M z-\zeta_{1}\right) /\left(M z-\zeta_{2}\right)=K\left(\left(z-\zeta_{1}\right) /\left(z-\zeta_{2}\right)\right)$. If $\zeta_{1}=\infty, K$ is defined by $M z-\zeta_{2}=K\left(z-\zeta_{2}\right)$. If $\zeta_{1}=\zeta_{2}$, then $K=1$. This $K$ is called the multiplier of $M$. Let $K=\rho e^{i \theta}$. For a matrix $M$ with real entries, there are three possibilities:

if $\rho=1, \theta \neq 0, M$ is called elliptic,

if $\rho \neq 1, \theta=0, M$ is called byperbolic,

if $\rho=1, \theta=0, M$ is called parabolic.

Received by the editors May 4, 1972 and, in revised form, October 2, 1972. $20 \mathrm{H} 10$.

AMS (MOS) subject classifications (1970). Primary 30A58, 10D15; Secondary 20H05,

Key words and phrases. Automorphic function, automorphic form, entire, discontinuous group. 
A parabolic of form $\pm\left(\begin{array}{ll}1 & b \\ 0 & 1\end{array}\right)$ is a translation. The multiplier for $M^{m}$ is $K^{m}$. A matrix $M$ has lft-order $n$ if its corresponding linear fractional transformation is of order $n$; i.e. $M^{n}= \pm l$. Only an elliptic matrix can have finite lft-order $n$, and then if and only if its multiplier $K$ is an $n$th root of unity [5, pp. 6, 7]. $\Gamma$ is called lft-cyclic if the corresponding linear fractional group is cyclic. Since both $\left(\begin{array}{ll}a & b \\ c & d\end{array}\right)$ and $\left(\begin{array}{ll}-a & -b \\ -c & -d\end{array}\right)$ correspond to the same linear fractional transformation, a normalized representative can be chosen such that $a \geq 0$, and if $a=0$ then $c>0$.

An ingenious classical result of Poincaré established that for most discontinuous groups, a corresponding nontrivial automorphic form of degree $\cdots r, r$ even and greater than two, is given by $\Sigma H(M z)(c z+d)^{-r}$ where $H(z)$ is a restricted type of rational function, and the sum is over all distinct bottom rows of $\mathrm{Mz}$ for normalized $M$ in $\Gamma[6]$. Elementary modifications and combinations of such functions give automorphic forms of any even degree for a discontinuous $\Gamma$. Such forms are merniorphic on the open upper half plane $\Pi^{+}$. A question raised by Marvin Knopf was: For what groups may a nonconstant entire automorphic form be found? The answer, incorporated in the results below, is: either the function is a polynomial (which case is then studied in full), or the group must be generated by at most two translations. Actually a more general approach was used, valid for functions meromorphic on the plane. For such a nonconstant $f$ with multiplier system identically one, we prove that either $\Gamma$ is finite or $\Gamma$ consists solely of translations, solely of nontranslational parabolic elements, solely of hyperbolic elements, solely of elliptic elements, or $\Gamma$ is the group extension of a hyperbolic group by a single elliptic. In each of these cases, $f(z)$ must have a particularly simple form; for instance if $\Gamma$ consists of parabolic elements (not translations), then $f(z)=A\left(z-z_{1}\right)^{\alpha}$ where $z_{1}$ is real, and the degree $r=\alpha \neq 0$. Results of Knopp are here useful. For multiplier systems not identically one, similar results are given.

Finally we study the case where $f$ is meromorphic on various other domains larger than the half plane. Rational functions fall naturally under the above-mentioned results. For nonrational functions:

(a) if $f$ is meromorphic on the whole plane, then the group $\Gamma$ is lft-cyclic and is generated by at most two translations;

(b) if $f$ is meromorphic in a domain containing the closed upper half plane, then additionally permitted in $\Gamma$ are elliptics of finite order (Theorem 7);

(c) if $f$ is meromorphic in a domain containing all except one point of the closed upper half plane, then additionally allowed in $\Gamma$ are hyperbolics with lower left entry zero and parabolics (Theorem 8);

(d) if $f$ is meromorphic in a domain containing all except two points of the closed upper half plane, then additionally permitted in $\Gamma$ are hyperbolics with arbitrary lower left entry (Theorem 9). 
Thus as the required domain of definition $\Omega$ of $f(z)$ shrinks, more freedom is allowed to the group $\Gamma$. One need not continue with theorems in which more points are removed, since already by $(d)$ above, $\Gamma$ may be cyclic of any type except that generated by an infinite order elliptic. That $\Gamma$ cannot contain an infinite order elliptic, even if $\Omega=\Pi^{+}$, follows from the proof of Theorem 7 . Thus Theorems 7 , 8 , and 9 fill the gap between Theorem 6 where $\Omega$ is the complex plane, and Poincaré's result where $\Omega$ is the open upper half plane. Theorems 7,8 and 9 are independent of whether or not a multiplier system is present.

Throughout, $a, b, c$, and $d$ are real and $a d-b c=1$. For $a, b, c$ and $d$ complex, see [3]. Until Theorem 10, $r$ is integral. In Theorems 1 through $6, v(M) \equiv 1$, $\forall M \in \Gamma$. C denotes the complex plane, and $\Pi^{+}$the open upper half plane.

\section{Functions meromorphic on $\mathrm{C}$.}

Theorem 1. Let f, an automorphic form of degree $r$ with respect to $\Gamma$, be meromorphic on the complex plane and be nonrational. Then $\Gamma$ contains at most translations, and is lft-cyclic, thus generated by at most two matrices.

Proof. Suppose $\Gamma$ contains $M=\left(\begin{array}{ll}a & b \\ c & d\end{array}\right)$ with $c \neq 0$. Set $\hat{f}(z)=f(M z)=$ $(c z+d)^{-r} f(z)$. $\hat{f}$ is meromorphic in a neighborhood of $M^{-1} \infty=-d / c$ so that $f$ is meromorphic at infinity. Since it is also meromorphic on all $\mathbf{C}, f(z)$ is rational, a contradiction. So $c=0$. Next it is shown $a=d= \pm 1$. If not, the two fixed points of $M$ are $\infty$ and $\zeta_{1}=b /(d-a)$. Let $f(z)=\sum_{n=N}^{\infty} a_{n}\left(z-\zeta_{1}\right)^{n}$ be the expansion valid in some deleted neighborhood of $\zeta_{1}$. Let $\gamma$ be a small circle about $\zeta_{1}$.

$$
\begin{aligned}
a_{k} & =\frac{1}{2 \pi i} \int_{\gamma} \frac{f(t)}{\left(t-\zeta_{1}\right)^{k+1}} d t=\frac{1}{2 \pi i} \int_{\gamma} \frac{(n t+d)^{r} f(M t)}{\left(t-\zeta_{1}\right)^{k+1}} d t \\
& =\frac{1}{2 \pi i} \int_{\gamma} \frac{d^{r} \sum_{n=N}^{\infty} a_{n}\left(((a t+b) / d)-\zeta_{1}\right)^{n}}{\left(t-\zeta_{1}\right)^{k+1}} d t \\
& =\frac{d^{r}}{2 \pi i} \int_{\gamma} \frac{\sum_{n=N}^{\infty} a_{n}\left((a t+b) / d-\left(a \zeta_{1}+b\right) / d\right)^{n}}{\left(t-\zeta_{1}\right)^{k+1}} d t \\
& =\frac{d^{r}}{2 \pi i} \int_{\gamma} \frac{\sum_{n=N}^{\infty} a_{n}(a / d)^{n}\left(t-\zeta_{1}\right)^{n}}{\left(t-\zeta_{1}\right)^{k+1}} d t .
\end{aligned}
$$

Here two facts have been utilized: first that $M$ carries a small neighborhood of $\zeta_{1}$ onto a small neighborhood of $\zeta_{1}$ and thus the substitution of $M t$ for $t$ in the expansion is a valid procedure for the determination of $f(M t)$, and second that $\zeta_{1}=\left(a \zeta_{1}+b\right) / d$. Now $\left|t-\zeta_{1}\right|$ remains greater than some nonzero constant for $t$ on $\gamma$, and $\sum_{n=N}^{\infty} a_{n}(a / d)^{n}\left(t-\zeta_{1}\right)^{n}$ converges absolutely and uniformly to $f(t) / d^{r}$ for $t$ on $\gamma$. Thus 


$$
\frac{\sum_{n=N}^{\infty} a_{n}(a / d)^{n}\left(t-\zeta_{1}\right)^{n}}{\left(t-\zeta_{1}\right)^{k+1}}
$$

converges likewise, and the interchange of order of summation and integration is justified.

$$
\begin{aligned}
a_{k} & =d^{r} \sum_{n=N}^{\infty} a_{n}\left(\frac{a}{d}\right)^{n} \frac{1}{2 \pi i} \int_{\gamma} \frac{\left(t-\zeta_{1}\right)^{n}}{\left(t-\zeta_{1}\right)^{k+1}} d t \\
& =d^{r}\left(\frac{a}{d}\right)^{k} a_{k}, \quad \text { since } \int_{\gamma}\left(t-\zeta_{1}\right)^{m} d t= \begin{cases}2 \pi i & \text { if } m=-1, \\
0 & \text { otherwise }\end{cases}
\end{aligned}
$$

So $a_{k}=a_{k} d^{r-2 k}$ since $c=0$ forces $a=d^{-1} . f(z)$ is nonrational so that $p$ and $q$ may be found such that $a_{p} \neq 0$ and $a_{q} \neq 0.1=d^{r-2 q}=d^{r-2 q}$. Not both $r-2 p$ and $r-2 q$ can be zero. Thus $|d|=1$ and since $a d-0 b=1, M= \pm\left(\begin{array}{ll}1 & b \\ 0 & 1\end{array}\right)$, a translation.

That $\Gamma$ is lft-cyclic is seen by the decomposition of $\Gamma$ into the disjoint union of $\Gamma^{\prime}$ and $\Gamma^{\prime \prime}$ where $\Gamma^{\prime}$ contains the matrices of form $\left(\begin{array}{ll}1 & b \\ 0 & 1\end{array}\right)$ and $\Gamma^{\prime \prime}$, not a group, those of form $\left(\begin{array}{cc}-1 & b \\ 0 & -1\end{array}\right)$. For $\Gamma^{\prime}, f(z)=(0 z+1)^{r} f(z+b)$ so there must be a $M_{0}$ in $\Gamma^{\prime}$ such that $\left|b_{0}\right|$ is minimal nonzero, for otherwise the values of $f$ would accumulate and $f$ would be constant. $\Gamma^{\prime}$ is generated by a $M_{0}$ by a euclidean algorithm type argument. If $M=\left(\begin{array}{cc}-1 & b \\ 0 & -1\end{array}\right), M^{2}=\left(\begin{array}{cc}1 & -2 b \\ 0 & 1\end{array}\right)$ so $b_{0}$ divides $2 b$ and $b=n b_{0} / 2$. If, for all such $b, n$ is even, $\Gamma$ is generated by $\left(\begin{array}{cc}1 & b_{0} \\ 0 & 1\end{array}\right)$ and $\left(\begin{array}{rr}-1 & b_{0} \\ 0 & -1\end{array}\right)$. If $n$ is odd for some $b, \Gamma$ is generated by $\left(\begin{array}{cc}-1 & b_{0} / 2 \\ 0 & -1\end{array}\right)$. In either case $\Gamma$ is $1 \mathrm{ft}$ cyclic.

Example. $f(z)=e^{2 \pi i z}, M=\left(\begin{array}{ll}1 & 1 \\ 0 & 1\end{array}\right)$.

Thcorem 2. Let

$$
f(z)=A \frac{\left(z-z_{1}\right)^{\alpha_{1}}\left(z-z_{2}\right)^{\alpha_{2}} \ldots\left(z-z_{m}\right)^{\alpha_{p}}}{\left(z-\zeta_{1}\right)^{\beta_{1}}\left(z-\zeta_{2}\right)^{\beta_{2}} \ldots\left(z-\zeta_{n}\right)^{\beta_{q}}}
$$

be an automorphic form of degree $r$ with respect to $\Gamma$, where $\alpha_{i}>0$ and $\beta_{i}>0$, and all of the $z_{i}$ and $\zeta_{j}$ are distinct. If $m+n>2$, then $\Gamma$ consists at most of elliptic matrices of order at most $2 \max (p, q)+2$ whose lft-order is at most $\max (p, q)+1$. If both $m$ and $n$ are greater than two, "max" may be replaced by "min".

Proof. The following claim is useful. Let $M=\left(\begin{array}{ll}a & b \\ c & d\end{array}\right) \in \Gamma$.

Claim. If $c \neq 0$ and $\left(\begin{array}{ll}a & b \\ c & d\end{array}\right)^{n}=\left(\begin{array}{ll}a_{n} & b_{n} \\ c_{n} & d_{n}\end{array}\right)$ and $\left(\begin{array}{ll}a & b \\ c & d\end{array}\right)^{m}=\left(\begin{array}{ll}a_{m} & b_{m} \\ s c_{n} & s d_{n}\end{array}\right)$, then $\left(\begin{array}{ll}a & b \\ c & d\end{array}\right)^{n}= \pm\left(\begin{array}{ll}a & b \\ c & d\end{array}\right)^{m}$. Without loss of generality $n<m$.

$$
z \stackrel{M}{\rightarrow} \frac{a z+b}{c z+d} \stackrel{M}{\rightarrow} \ldots \stackrel{M}{\rightarrow} \frac{a_{n} z+b_{n}}{c_{n} z+d_{n}} \stackrel{M}{\rightarrow} \ldots-\stackrel{M}{\rightarrow} \frac{a_{m} z+b_{m}}{s c_{n} z+s d_{n}} \stackrel{M}{\rightarrow} \ldots
$$


Let $z=-d_{n} / c_{n}$. Then $-d_{n} / c_{n} \stackrel{M}{\rightarrow} \cdots \stackrel{M}{\rightarrow} \infty \stackrel{M}{\rightarrow} \cdots \stackrel{M}{\rightarrow} \infty \stackrel{M}{\rightarrow} \cdots$, so that $M^{m-n}$ has $\infty$ as a fixed point in addition to the two finite fixed points which $M$ possesses. Thus $M^{m-n}= \pm I$ and the claim follows.

Since $p+q>2, w_{0}$, a zero or pole of $f$, can be found which is neither of the fixed points of $M$. Assume it is a zero. $f\left(w_{0}\right)=0=\left(c_{m} w_{0}+d_{m}\right)^{r} f\left(M^{m} w_{0}\right)$. If $c_{m} w_{0}+d_{m}=0=c_{n} w_{0}+d_{n}$ for $m \neq n$, then (since neither $c_{m}$ nor $c_{n}$ can be zero because the determinants must be one) $-d_{m} / c_{m}=w_{0}=-d_{n} / c_{n}$, so that $c_{m}=s c_{n}$ and $d_{m}=s d_{n}$ where $s=-c_{m} w_{0} / d_{n}$. By the claim, $M^{n}= \pm M^{m}$ and $M^{n-m}= \pm I$ so $M$ is of finite order elliptic type. Thus remaining to be considered is the case of $c_{m} w_{0}+d_{m}=0$ for at most two $m$. The $f\left(M^{m} w_{0}\right)$ must now be zero for every $m$ but two. Unless $M$ is of finite order elliptic, the $M^{m}$ would all be distinct which would imply $f$ has infinitely many zeros, a contradiction. The same reasoning applies if $w_{0}$ is a pole.

If $w_{0}$ is a zero, a count of the number of times $\left(c_{m} w_{0}+d_{m}\right)^{r} f\left(M^{m} w_{0}\right)$ may be zero $(m=0,1,2, \cdots)$ yields twice for each zero of $f$ and twice more for the possibility $\left(c_{m} w_{0}+d_{m}\right)=0$, i.e., $M^{m} w_{0}=\infty$, so that the maximum order is $2 p+2$. If $w_{0}$ is a pole, the maximum order is $2 q+2$. If both $p$ and $q$ are greater than two, then $w_{0}$ can be chosen arbitrarily as either a zero or a pole. Thus the given bounds on the magnitude of the order are valid.

Example. $f(z)=(z-1)^{2}(z+4 / 3)^{2}(z+5 / 2)^{2} /(z-(-3+i \sqrt{ } 3) / 2)^{3}, M=$ $\left(\begin{array}{rr}1 & 3 \\ -1 & -2\end{array}\right), M^{3}=1, r=-1$.

Under the hypotheses of Theorem 2, $\Gamma$ is finite by Burnside's theorem [1, p. $251]$ since $\Gamma$ is periodic of period at $\operatorname{most}(2 \max (p, q)+2)$ !

Theorem 3. Suppose $q(z)=A\left(z-z_{1}\right)^{a} /\left(z-\zeta_{1}\right)^{\beta}$ is an automorpbic form of degree $r$ on $\Gamma$ where $z_{1} \neq \zeta_{1}, a>0$ and $\beta>0$. Then

\begin{tabular}{l|l|l} 
if $r$ & $z_{1}$ and $\zeta_{1}$ & \multicolumn{1}{|c}{ then $\Gamma$ consists of } \\
\hline (a) $r=a-2 \beta$ & nonreal-real & $\begin{array}{l}\text { elliptics of lft-order at most two fixing } z_{1} \text { and } \\
\text { interchanging } \zeta_{1} \text { and } \infty\end{array}$ \\
(b) $r=2 \alpha-\beta$ & real-nonreal & $\begin{array}{l}\text { elliptics of lft-order two fixing } \zeta_{1} \text { and inter- } \\
\text { changing } z_{1} \text { and } \infty\end{array}$ \\
(c) $r=\alpha-\beta$ & nonreal $z_{1}=\bar{\zeta}_{1}$ & $\begin{array}{l}\text { elliptics fixing } z_{1} \text { and } \zeta_{1} \text { of lft-order at most } \\
1 / 2(\alpha+\beta) \text { if } \alpha+\beta \text { is even, of lft-order at } \\
\text { most }(\alpha+\beta) \text { if } \alpha+\beta \text { is odd. }\end{array}$
\end{tabular}

Otherwise $\Gamma=\{I\}$ or $\{I,-I\}$. 
Also in case (a), $a=2 \beta+4 n$ for some integer $n$; in case (b), $\beta=2 \alpha+4 n$ for some integer $n$.

Proof. If $c=0$ for some $M=\left(\begin{array}{ll}a & b \\ c & d\end{array}\right) \in \Gamma$, then $\infty$ is a fixed point of $M$, so at least one of $z_{1}$ and $\zeta_{1}$ is not. Assume $z_{1}$ is not a fixed point. Then $0=f\left(z_{1}\right)$ $=d^{r} f\left(M z_{1}\right)$ so that $M z_{1}$ is also a zero of $f$. But $M z_{1} \neq z_{1}$ and $M z_{1} \neq \infty$. This contradicts the form of $f$. Likewise if $\zeta_{1}$ is not a fixed point, then $f$ must have a pole at $M \zeta_{1}$, again a contradiction. So $c \neq 0$.

$$
f(z)=A \frac{\left(z-z_{1}\right)^{a}}{\left(z-\zeta_{1}\right)^{\beta}}=(c z+d)^{r} f(M z)=A(c z+d)^{r-a_{+} \beta} \frac{\left(z\left(a-c z_{1}\right)+b-d z_{1}\right)^{\alpha}}{\left(z\left(a-c \zeta_{1}\right)+b-d \zeta_{1}\right)^{\beta}} .
$$

$$
\left(z-z_{1}\right)^{a}\left(z\left(a-c \zeta_{1}\right)+b-d \zeta_{1}\right)^{\beta}
$$

$$
=c^{r-a+\beta}\left(z+d !^{\prime} c\right)^{r-a+\beta}\left(z-\zeta_{1}\right)^{\beta}\left(z\left(a-c z_{1}\right)+b-d z_{1}\right)^{\beta} \text {. }
$$

Case 1. $a-c \zeta_{1}=0$. Then $a-c z_{1} \neq 0$ since $z_{1} \neq \zeta_{1}$ and $c \neq 0$. (*) becomes

$$
\begin{aligned}
& \left(z-z_{1}\right)^{a}\left(b-d \zeta_{1}\right)^{\beta}=c^{r-a+\beta}(z+d / c)^{r-a+\beta}\left(z-\zeta_{1}\right)^{\beta}\left(z\left(a-c z_{1}\right)+b-d z_{1}\right)^{a} \\
& =c^{r-\alpha+\beta}\left(a-c z_{1}\right)^{\alpha}(z+d / c)^{r-\alpha+\beta}\left(z-\zeta_{1}\right)^{\beta}\left(z+\left(b-d z_{1}\right) /\left(a-c z_{1}\right)\right)^{\alpha} \\
& =c^{r-a+\beta}\left(a-c z_{1}\right)^{a}(z+d / c)^{r-a+\beta}\left(z-\zeta_{1}\right)^{\beta}\left(z-M^{-1} z_{1}\right)^{a} \text {. }
\end{aligned}
$$

So either $M^{-1} z_{1}=-d / c$ (but this implies $z_{1}=\infty$ ), or $M^{-1} z_{1}=z_{1}$ and $\zeta_{1}=-d / c$ and $-\beta=r-\alpha+\beta$. So $z_{1}$ is fixed and $\zeta_{1}=-d / c \stackrel{M}{\rightarrow} \infty \stackrel{M}{\rightarrow} a / c=\zeta_{1}$ so that $M^{2}= \pm I$. This is (a). Also $\left(b-d \zeta_{1}\right)^{\beta}=c^{r-a+\beta}\left(a-c z_{1}\right)^{\alpha}$ and $\left(b-d \zeta_{1}\right)^{\beta}=$ $(b-d a / c)^{\beta}=(-1 / c)^{\beta}$. But $a=-d$ so that $M z_{1}=z_{1}$ has solution $z_{1}=(a \pm i) / c$ and $\left(a-c z_{1}\right)=\mp i$. Thus $(\mp i)^{\alpha}=(-1)^{\beta}$, which implies $\alpha=2 \beta+4 n$ for some integer $n$.

Case 2. $a-c z_{1}=0$. Then $a-c \zeta_{1} \neq 0$ since $z_{1} \neq \zeta_{1}$ and $c \neq 0$. (*) becomes

$$
\left(z-z_{1}\right)^{a}\left(z\left(a-c \zeta_{1}\right)+\left(b-d \zeta_{1}\right)\right)^{\beta}=c^{r-a+\beta}(z+d / c)^{r-a+\beta}\left(z-\zeta_{1}\right)^{\beta}\left(b-d z_{1}\right)^{a} \text {. }
$$

Thus $z_{1}=-d / c$ and $\zeta_{1}=M^{-1} \zeta_{2}$ and $\alpha=r-\alpha+\beta$. Thus $z_{1}=-d / c \stackrel{M}{\rightarrow} \infty$ $\stackrel{M}{\rightarrow} a / c=z_{1}$ so that $M^{2}= \pm I, \zeta_{1}$ is fixed, and $r=2 \alpha-\beta$. This is (b). That $\beta=2 \alpha+4 n$ follows as in Case 1 .

Case 3. $a-c z_{1} \neq 0$ and $a-c \zeta_{1} \neq 0$. (*) becomes

$$
\left(z-z_{1}\right)^{\alpha}\left(a-c \zeta_{1}\right)^{\beta}\left(z-M^{-1} \zeta_{1}\right)^{\beta}=c^{r-\alpha+\beta}(z+d / c)^{r-a+\beta}\left(z-\zeta_{1}\right)^{\beta}\left(a-c z_{1}\right)^{\alpha}\left(z-M^{-1} z_{1}\right)^{\alpha} \text {. }
$$

The two sides must be of the same degree in $z$; hence $r-\alpha+\beta=0$. Also 
$z_{1}=M^{-1} z_{1}$ and $\zeta_{1}=M^{-1} \zeta_{1}$ because $z_{1} \not \zeta_{1}$. Necessarily $\left(a-c z_{1}\right)^{\alpha}=$ $\left(a-c \zeta_{1}\right)^{\beta}$. If $K$ is defined by $\left(M z-z_{1}\right) /\left(M z-\zeta_{1}\right)=K\left(z-z_{1}\right) /\left(z-\zeta_{1}\right)$ where $z_{1}$ and $\zeta_{1}$ are the fixed points of $M_{2}$ then

$$
M z-z_{1}=K\left[M z\left(\frac{z-z_{1}}{z-\zeta_{1}}\right)-\zeta_{1}\left(\frac{z-z_{1}}{z-\zeta_{1}}\right)\right]
$$

so

$M z\left[1-K\left(\frac{z-z_{1}}{z-\zeta_{1}}\right)\right]=z_{1}-K \zeta_{1}\left(\frac{z-z_{1}}{z-\zeta_{1}}\right)$ and $M z=\frac{z\left(z_{1}-K \zeta_{1}\right)+\left(K z_{1} \zeta_{1}-z_{1} \zeta_{1}\right)}{z(1-K)+\left(K z_{1}-\zeta_{1}\right)}$.

But $M=\left(\begin{array}{ll}a & b \\ c & d\end{array}\right)$ where $a d-b c=1$. Note that if we select $S=\sqrt{K}\left(z_{1}-\zeta_{1}\right)$ then

$$
M z=\frac{z\left(z_{1}-K \zeta_{1}\right) / S+(K-1) z_{1} \zeta_{1} / S}{z(1-K) / S+\left(K z_{1}-\zeta_{1}\right) / S}
$$

and the corresponding matrix has determinant one. Thus

$$
a=\frac{z_{1}-\zeta_{1} K}{\sqrt{K}\left(z_{1}-\zeta_{1}\right)}, \quad b=\frac{(K-1) z_{1} \zeta_{1}}{\sqrt{K}\left(z_{1}-\zeta_{1}\right)}, \quad c=\frac{1-K}{\sqrt{K}\left(z_{1}-\zeta_{1}\right)}, \quad d=\frac{K z_{1}-\zeta_{1}}{\sqrt{K}\left(z_{1}-\zeta_{1}\right)}
$$

and the equation $\left(a-c z_{1}\right)^{\alpha}=\left(a-c \zeta_{1}\right)^{\beta}$ becomes

$$
\left[\frac{z_{1}-K \zeta_{1}}{\sqrt{K}\left(z_{1}-\zeta_{1}\right)}-\frac{(1-K) z_{1}}{\sqrt{K}\left(z_{1}-\zeta_{1}\right)}\right]^{\alpha}=\left[\frac{z_{1}-K \zeta_{1}}{\sqrt{K}\left(z_{1}-\zeta_{1}\right)}-\frac{(1-K) \zeta_{1}}{\sqrt{K}\left(z_{1}-\zeta_{1}\right)}\right]^{\beta},
$$

$(1 / \sqrt{K})^{a}=(\sqrt{K})^{\beta}$. So $(\sqrt{K})^{\alpha+\beta}=1$. Case (c) follows. $z_{1}=\bar{\zeta}_{1}$ because $z_{1}$ and $\zeta_{1}$ are the fixed points of a finite order elliptic.

Examples. (a) $f(z)=(z-(1+i))^{2} /(z-1), M=\left(\begin{array}{ll}1 & -2 \\ 1 & -1\end{array}\right)$,

(b) $f(z)=(z-1) /(z-(1+i))^{2}, M=\left(\begin{array}{ll}1 & -2 \\ 1 & -1\end{array}\right)$,

(c) $f(z)=(z-(1+i))^{2} /(z-(1-i))^{2}, M=\left(\begin{array}{cc}1 & -2 \\ 1 & -1\end{array}\right)$.

Under the hypotheses of Theorem $3, \Gamma$ is finite. In case (a) suppose $\Gamma$ contains two matrices $M_{1}$ and $M_{2}$. Then $M_{1} M_{2}$ must also satisfy the conditions on (a), but it fixes $\zeta_{1}$. Thus $\Gamma=\{M, l\}$ at most. Likewise in case (b). In case (c) $\Gamma$ is finite by an application of Burnside's theorem [1, p. 251].

Theorem 4. (Knopp [4, pp. 513-515]). Let $p(z)=A\left(z-z_{1}\right)^{a_{1}}\left(z-z_{2}\right)^{a_{2}}$ be an automorphic form of degree $r$ on $\Gamma$ where $z_{1} \neq z_{2}$ and $\alpha_{1}>0, \alpha_{2}>0$. Then 


\begin{tabular}{|c|c|c|}
\hline if $r$ & $z_{1}, z_{2}$ & then $\Gamma$ consists of \\
\hline (a) $r=2 \alpha_{1}=2 \alpha_{2}$ & $z_{1}, z_{2}$ real & $\begin{array}{l}\text { byperbolics fixing } z_{1} \text { and } z_{2} \text {; } \\
\text { elliptics of lft-order two interchanging } \\
z_{1} \text { and } z_{2} \text { with } \alpha_{1}, \alpha_{2} \text { even }\end{array}$ \\
\hline (b) $r=2 \alpha_{1}=2 \alpha_{2}$ & $z_{1}=\bar{z}_{2}$, nonreal & elliptics fixing $z_{1}$ and $z_{2}$ \\
\hline (c) $r=2 \alpha_{1}=2 a_{2}$ & $\begin{array}{l}z_{1}, z_{2} \in \Pi^{+} \\
\text {or } \bar{z}_{1}, \bar{z}_{2} \in \Pi^{+}\end{array}$ & $\begin{array}{l}\text { elliptics of lft-order two interchang- } \\
\text { ing } z_{1} \text { and } z_{2} \text { with } \alpha_{1}, \alpha_{2} \text { even }\end{array}$ \\
\hline (d) $r=3 a_{1}=3 a_{2}$ & $z_{1}, z_{2}$ real & $\begin{array}{l}\text { elliptics of lft-order three permuting } \\
z_{1}, z_{2} \text { and } \infty\end{array}$ \\
\hline (e) $r=a_{1}+a_{2} \neq 2 a_{1}$ & $z_{1}=\bar{z}_{2}$ & $\begin{array}{l}\text { elliptics fixing } z_{1} \text { and } z_{2} \text { of lft- } \\
\text { order at most } r-2 \alpha_{2} \text { if } r \text { is odd; } \\
r / 2-\alpha_{2} \text { if } r \text { is even }\end{array}$ \\
\hline (f) $r=2 \alpha_{1}+a_{2}$ & $\begin{array}{ll}z_{1} & \text { real } \\
z_{2} & \text { nonreal }\end{array}$ & $\begin{array}{l}\text { elliptics of lft-order two fixing } z_{2} \\
\text { and interchanging } z_{1} \text { and } \infty\end{array}$ \\
\hline (g) $r=2 \alpha_{2}+\alpha_{1}$ & $\begin{array}{l}z_{2} \text { real, } \\
z_{1} \text { nonreal }\end{array}$ & $\begin{array}{l}\text { elliptics of lft-order two fixing } z_{1} \\
\text { and interchanging } z_{2} \text { and } \infty\end{array}$ \\
\hline
\end{tabular}

Otherwise $\Gamma=\{I\}$ or $\{I,-I\}$.

\section{Examples.}

(a) (1) $p(z)=(z-1)(z+1), M=1 / 4\left(\begin{array}{rr}5 & -3 \\ -3 & 5\end{array}\right)$, (2) $p(z)=(z-2)^{2}(z+3)^{2}, M=1 / 2\left(\begin{array}{cc}1 & -5 \\ 1 & -1\end{array}\right)$,

(b) $p(z)=(z-i)(z+i), M=\left(\begin{array}{cc}\cos \theta & -\sin \theta \\ \sin \theta & \cos \theta\end{array}\right)$, where if $\theta=2 \pi m / n$ then $M$ is of order $n$, and if $\theta / 2 \pi$ is irrational then $M$ is of infinite order,

(c) $p(z)=(z-i)^{2}(z-2 i)^{2}, M=\left(\begin{array}{c}0 \\ 1 / \sqrt{ } 2\end{array}-\sqrt{2}\right)$,

(d) $p(z)=(z-1)(z-2), M=\left(\begin{array}{ll}1 & -3 \\ 1 & -2\end{array}\right)$,

(e) $p(z)=(z-i)^{5}(z+i), M=\left(\begin{array}{rr}0 & 1 \\ -1 & 0\end{array}\right)$,

(f) $p(z)=(z-1)(z-(1+i))^{2}, M=\left(\begin{array}{ll}1 & -2 \\ 1 & -1\end{array}\right)$,

(g) reversed order of factors in (f).

Pertinent to the question of what types of groups are permitted when an automorphic form of a certain type is given, is the size of $\Gamma$ in (a) through $(g)$. Elementary considerations show in (c), (f), and $(g), \Gamma$ is at most $\{ \pm I, \pm M\}$ where $M^{2}= \pm l$. In (d), $\Gamma$ is at most $\left\{ \pm l, \pm M, \pm M^{2}\right\}$ where $M^{3}= \pm l$. In (e), as in Theorem 3, $\Gamma$ is also finite by Burnside's theorem. However in (a), $\Gamma$ could in fact be all matrices which satisfy either condition. Clearly every elliptic in (a) satisfies $M^{2}= \pm I$ since $M^{2}$ has four fixed points. Thus if $\Gamma$ is not finite, either case (a) or (b) must hold. 
Theorem 5 (Knopp [4, pp. 515, 516])。 Let $p(z)=A\left(z-z_{1}\right)^{\alpha}$ be an automorphic form of degree $r$ on $\Gamma$ where $a>0$. Then

\begin{tabular}{l|l|l}
\multicolumn{1}{c|}{ if $r$} & and $z_{1}$ & \multicolumn{1}{c}{ then $\Gamma$ consists of } \\
\hline (a) $r=2 \alpha$ & $z_{1}$ real & $\begin{array}{l}\text { byperbolics fixing } z_{1} \text { and } \infty \text {; and elliptics of lft- } \\
\text { order two intercbanging } z_{1} \text { and } \infty \text {, and a even }\end{array}$ \\
(b) $r=a$ & $z_{1}$ real & parabolics fixing $z_{1}$ \\
(c) $r=a$ & $z_{1}$ nonreal & $\begin{array}{l}\text { elliptics fixing } z_{1} \text { of lft-order } r / 2 \text { if } r \text { is even, } \\
\text { and lft-order } r \text { if } r \text { is odd }\end{array}$
\end{tabular}

Otherwise $\Gamma=\{I\}$ or $\{I .-I\}$.

Examples.
(a) (1) $p(z)=(z+2 / 3), M=\left(\begin{array}{cc}2 & 1 \\ 0 & 1 / 2\end{array}\right)$,
(2) $p(z)=(z-1)^{2}, M=\left(\begin{array}{ll}1 & -2 \\ 1 & -1\end{array}\right)$,

(b) $p(z)=(z-1)^{3}, M=\left(\begin{array}{cc}3 / 2 & -1 / 2 \\ 1 / 2 & 1 / 2\end{array}\right)$,

(c) $p(z)=(z-(3+i \sqrt{ } 3) / 2)^{3}, M=\left(\begin{array}{ll}1 & -\frac{3}{2} \\ 1 & -2\end{array}\right)$.

$\Gamma$ in (a) could in fact be all matrices which satisfy either condition. If $M$ is elliptic, $M^{2}$ has four fixed points so $M^{2}= \pm I$. In (c) $\Gamma$ is finite by Burnside's theorem.

Theorem 6 (Summary). Let $f(z)$, nonconstant, meromorpbic in $\mathrm{C}$, be an automorpbic form of degree $r$ on $\Gamma$ with multiplier system identically one.

(1) If $f(z)$ is not the quotient of two polynomials, then $\Gamma$ admits at most translations, and is lft-cyclic. (Theorem 1$)$.

(2) If $f(z)=A\left(z-z_{1}\right)^{a_{1}}\left(z-z_{2}\right)^{a_{2}} \ldots\left(z-z_{n}\right)^{a_{n}} /\left[\left(z-\zeta_{1}\right)^{\beta_{1}}\left(z-\zeta_{2}\right)^{\beta_{2}} \ldots\right.$ $\left.\left(z-\zeta_{m}\right)^{\beta_{m}}\right]$ where the $z_{i}$ and the $\zeta_{j}$ are all distinct and $\alpha_{i}>0, \beta_{j}>0$, and

if $m=0, n=1$, then Theorem 5 lists the possibilities for $\Gamma$. $m=0, n=2$, then Theorem 4 lists the possibilities for $\Gamma$. $m=1, n=1$, then Theorem 3 lists the possibilities for $\Gamma$. $m=1, n=0$, then the results are as in Theorem 5 . $m=2, n=0$, then the results are as in Theorem 4 .

Otherwise, $\Gamma$ contains at most finite order elliptics as seen by Theorem 2 .

Proof. The only parts which are not already proved are the cases $m=1,2$ with $n=0$. However if $f(z)$ is an automorphic form of degree $r$, then $1 / f(z)$ is an automorphic form of degree - r. In $1 / f(z)$, the numerator has 1 or 2 distinct zeros and the denominator no zeros, which was handled in Theorems 4 and 5 .

A summary of the previous results on the finiteness of $\Gamma$ in the above theorem is: for $f$ meromorphic in $\mathbf{C}_{2}$ and an automorphic form of degree $r$ on $\Gamma, \Gamma$ is 
finite except in the following few cases:

(a) $f(z)$ is constant. If $r=0, \Gamma$ is arbitrary. If $r \neq 0, \Gamma$ can contain only translations.

(b) $f(z)$ nonrational, $\Gamma$ lft-cyclic containing only translations. $\Gamma$ is thus discontinuous.

(c) $f(z)=A\left(z-z_{1}\right)^{r}, z_{1}$ real, $r \neq 0, \Gamma$ consists solely of parabolics fixing $z_{1}$. By Theorems $2 \mathrm{~F}$ and $2 \mathrm{H}$ of $[5, \mathrm{Pp} .13,15], \Gamma$ is discontinuous if and only if $\Gamma$ is lft-cyclic in this case.

(d) $f(z)=A\left(z-z_{1}\right)^{r / 2}, z_{1}$ real, $0 \neq r$ even, $\Gamma$ consists of hyperbolics fixing $z_{1}$ and $\infty$. If $r / 2$ is even, also permitted are elliptics of lft-order two which interchange $z_{1}$ and $\infty$. If $\Gamma^{\prime}$ is the subgroup of all hyperbolics in $\Gamma$, and $E$ and $E^{\prime}$ are elliptics in $\Gamma$, examination of the fixed points shows $E^{-1} E^{\prime} \in \Gamma^{\prime}$ so $E^{\prime} \in E \Gamma^{\prime}$. Thus $\Gamma=\Gamma^{\prime} \cup E \Gamma^{\prime}$ and as in (c) is discontinuous if and only if $\Gamma^{\prime}$ is lft-cyclic. So $\Gamma$ is discontinuous if and only if $\Gamma$ is lft-generated by a hyperbolic, or if $r / 2$ is even, by at most a hyperbolic and an elliptic.

(e) $f(z)=A\left(z-z_{1}\right)^{r / 2}\left(z-z_{2}\right)^{r / 2}, z_{1}, z_{2}$ real, $0 \neq r$ even, $\Gamma$ consists of hyperbolics fixing $z_{1}$ and $z_{2}$. If $r / 2$ is even, also permitted are elliptics of lftorder two which interchange $z_{1}$ and $z_{2}$. As in (d), if $\Gamma$ is discontinuous, $\Gamma$ is lft-generated by a hyperbolic, or if $r / 2$ is even, by at most a hyperbolic and an elliptic.

(f) $f(z)=A\left(z-z_{1}\right)^{r / 2}\left(z-\bar{z}_{1}\right)^{r / 2}, z_{1}$ not real, $0 \neq r$ even, $\Gamma$ consists of elliptics fixing $z_{1}$ and $\bar{z}_{1}$. Since $\Gamma$ is assumed nonfinite, there are elliptics of arbitrarily high order. Since each $M$ can be considered a rotation about the circle with hyperbolic center $z_{1}$, and since $\left\{M^{i} z\right\}_{i=1}^{\infty}$ is $n$ points where $n$ is the order of $M$, necessarily $\Gamma z$ accumulates for a nonfixed $z$. By Theorem $2 F$ of $[5$, p. 13], $\Gamma$ is not discontinuous.

III. Multiplier systems. If $v(M) f(z)=(c z+d)^{r} f(M z)$, then Theorems 1 through 6 easily generalize in each of the two cases: $|\nu(M)|=1 \forall M \in \Gamma$, and $v(M)$ general. The proofs are merely reproductions of those already given with $v(M)$ inserted in the proper places, and are therefore omitted.

Theorems 1 and 2 obviously remain valid.

Theorem. Suppose $f(z)=A\left(z-z_{1}\right)^{\alpha} /\left(z-\zeta_{1}\right)^{\beta}$ is an automorpbic form of degree $r$ with respect to $\Gamma$ and has multiplier system $v(M)$.

3(i) $|v(M)|=1 \forall M \in \Gamma$ then additionally permitted to the results of Theorem 4 3 is $r=\alpha-\beta, z_{1}$ nonreal and $z_{1}=\bar{\zeta}_{1}, M$ elliptic fixing $z_{1}$ and $\zeta_{1}$ where $v(M)=K^{(\alpha+\beta) / 2}$.

3(ii) If $v(M)$ is general, then additionally permitted is $r=\alpha-\beta, z_{1}$ real, $z_{2}$ real, $M$ byperbolic fixing $z_{1}$ and $\zeta_{1}$, with $v(M)=K^{(\alpha+\beta) / 2}$. 
Also the notes $\alpha=2 \beta+4 n$ in case (a) and $\beta=2 \alpha+4 n$ in case (b) must be dropped.

Theorem. Suppose $f(z)=A\left(z-z_{1}\right)^{a_{1}}\left(z-z_{2}\right)^{a_{2}}$ is an automorphic form of degree $r$ with respect to $\Gamma$ and bas multiplier system $v(M)$.

4(i) If $|v(M)|=1 \forall M \in \Gamma$, then additionally allowed to the results of Theorem 4 is $r=a_{1}+a_{2}, z_{1}=\bar{z}_{2}$ nonreal, $M$ elliptic fixing $z_{1}$ and $z_{2}$. Also if $v(M)=$ $e^{2 \pi i \phi}$ and $K=e^{2 \pi i \theta}$ then $\phi \equiv \theta\left(r / 2-\alpha_{2}\right)$ modulo 1 .

4(ii) If $v(M)$ is general, then additionally permitted is $r=\alpha_{1}+\alpha_{2}, z_{1}$ real, $z_{2}$ real, $M$ byperbolic fixing $z_{1}$ and $z_{2}$ with $v(M)=K^{(r / 2)-a_{2}}$ real, positive, not 1 .

Theorem. Suppose $f(z)=A\left(z-z_{1}\right)^{\alpha}$ is an automorpbic form of degree $r$ with respect to $\Gamma$ and has multiplier system $v(M)$.

5(i) If $|v(M)|=1 \forall M \in \Gamma$, then additionally allowed to the results of Theorem 5 is $r=\alpha, z_{1}$ nonreal, $M$ elliptic fixing $z_{1}$. If $v(M)=e^{2 \pi i \phi}$ and $K=e^{2 \pi i \theta}$, then $\theta \equiv \phi \alpha / 2$ modulo 1 .

5(ii) If $v(M)$ is general, then additionally permitted are

(a) $r=a, z_{1}$ real, $M$ byperbolic fixing $z_{1}$, with $v(M)=K^{r / 2}$.

(b) $r \neq 2 \alpha, z_{1}$ real, $M$ byperbolic fixing $z_{1}$ and $\infty$ with $v(M)=a^{2 a_{-r}}$

$=K^{a_{-r} / 2}$. Here a denotes the upper left entry of $M$.

Thus the summary theorem, Theorem 6, can be extended to the two cases: $|v(M)|=1 \forall M \in \Gamma$, and $v(M)$ general, by the replacement of 3,4 , and 5 by $3(i)$, 4(i), and 5(i), and 3(ii), 4(ii), and 5(ii) respectively. The few possibilities of functions automorphic with respect to infinite groups which also have $v(M)=1$ $\forall M \in \Gamma$ are listed in (a)-(f) following Theorem 6. If the condition $v(M)=1$ $\forall M \in \Gamma$ is replaced by $|v(M)|=1 \forall M \in \Gamma$, then additionally permitted for infinite $\Gamma$ are

(g) $f(z)=A\left(z-z_{1}\right)^{a}\left(z-\bar{z}_{1}\right)^{\beta}, z_{1}$ nonreal, $\alpha \beta>0, r=\alpha+\beta, \Gamma$ consists solely of elliptics fixing $z_{1}$ and $\bar{z}_{1}$. As in (f), $\Gamma$ is not discontinuous.

(h) $f(z)=A\left(z-z_{1}\right)^{r}, z_{1}$ nonreal, $r \neq 0, \Gamma$ consists solely of elliptics fixing $z_{1}$ and $\bar{z}_{1}$. As above $\Gamma$ is not discontinuous.

(i) $f(z)=A\left(z-z_{1}\right)^{\alpha} /\left(z-\bar{z}_{1}\right)^{\beta}, z_{1}$ nonreal, $\alpha \beta>0, r=\alpha-\beta, \Gamma$ consists solely of elliptics fixing $z_{1}$ and $\bar{z}_{1}$. As above, $\Gamma$ is not discontinuous.

If $v(M)$ is general, then in addition to the above, the following nonfinite $\Gamma$ are allowed:

(j) $f(z)=A\left(z-z_{1}\right)^{\alpha}\left(z-z_{2}\right)^{\beta}, z_{1}, z_{2}$ real, $\alpha \beta>0, \alpha \neq \beta, r=\alpha+\beta, \Gamma$ consists solely of hyperbolics fixing $z_{1}$ and $z_{2}$. As in (b) $\Gamma$ is discontinuous if and only if $\Gamma$ is lft-cyclic. 
(k) $f(z)=A\left(z-z_{1}\right)^{r}, z_{1}$ real, $r \neq 0$. Note that this is an addition to (c) above. $\Gamma$ may now also include hyperbolics which fix $z_{1}$. But if $\Gamma$ is discontinuous, then by Theorems $2 \mathrm{I}$ and $2 \mathrm{H}$ of [5, p. 15], $\Gamma$ is Ift-cyclic.

(l) $f(z)=A\left(z-z_{1}\right)^{\alpha}, z_{1}$ real, $0 \neq \alpha \neq r \neq 2 \alpha, r \neq 0, \Gamma$ consists of hyperbolics fixing $z_{1}$ and $\infty$. As in (k) if $\Gamma$ is discontinuous, $\Gamma$ is lft-cyclic.

(m) $f(z)=A\left(z-z_{1}\right)^{\alpha} /\left(z-z_{2}\right)^{\beta}, z_{1}, z_{2}$ real, $\alpha \beta>0, r=\alpha-\beta, \Gamma$ consists of hyperbolics fixing $z_{1}$ and $z_{2}$. As above Ift-cyclic is a characterization of such $\Gamma$ which are discontinuous.

Thus for nonconstant $f$, if $\Gamma$ is discontinuous, either $\Gamma$ is finite or lftcyclic except in the two cases (c) and (d).

The study of automorphic forms meromorphic on the complex plane is completed.

גV. Large domains. Next to be considered are automorphic forms meromorphic on $\Omega$ where $\Pi^{+} \subset \Omega \subset \mathbf{C}$.

Theorem 7. Suppose $f(z)$ is an automorpbic form of degree $r$ with multiplier system $v(M)$, and is meromorphic in an open set $\Omega$ containing $\Pi^{+}$, the upper half plane. Then the conclusions of Theorem 6 and its generalizations (paragraph following Theorems 5(i), 5(ii)) are valid, with the sole exception that if $f(z)$ is not the quotient of polynomials (Case 1) then also allowed in $\Gamma$ are elliptics of finite order.

Proof. Case 1 of Theorem 6 is of course the only concern.

Parabolics, $c=0$ is permitted in Theorem 6. If $c \neq 0$, the fixed point of $M=\left(\begin{array}{ll}a & b \\ c & d\end{array}\right)$, parabolic, is real. In Figure 1, any one shaded region (or any one unshaded region) forms a fundamental region for the group generated by $M$. Since $\overline{\Pi^{+}} \subset \Omega$, we may choose a fundamental region lying inside $\Omega$. Thus $f(z)$

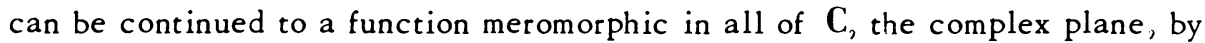
use of the functional equation $f(z)=v(M)^{-1}(c z+d)^{r} f(M z)$. Theorem 6 may then be applied. See also [2, pp. 21-23].

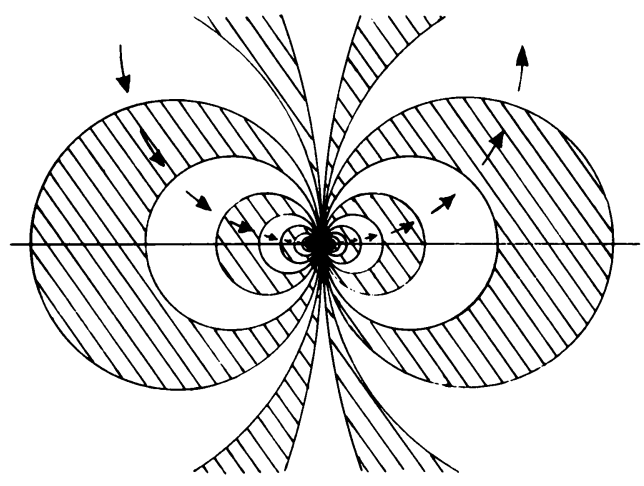

Figure 1 
Hyperbolics. The same analysis is valid as in the above case, since in either the $c=0$ or $c \neq 0$ cases, we may choose fundamental regions within $\Omega$, and then extend $f(z)$ by the functional equation to be meromorphic on all of $\mathbf{C}$. Any single shaded region is a fundamental region [2, pp. 18, 19]. See Figure 2.

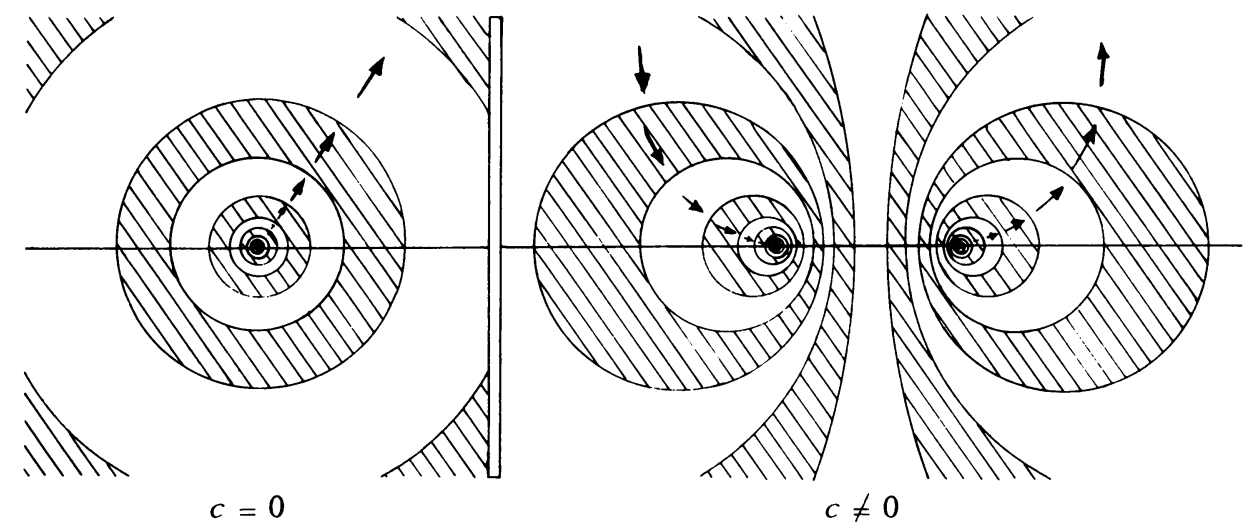

Figure 2

Elliptics of infinite order. Suppose $r=0$. A sequence $\{k(i)\}$ can be found so that if $z_{1} \in \Pi^{+}$is not a fixed point, then $\left\{M^{k(i)} z_{1}\right\}$ converges to a point in $\Pi^{+}$ [5, p. 12]. But $f(z)=1 f\left(M^{k(i)} z_{1}\right)$, so that the identity theorem implies $f(z)$ is constant and thus rational.

If $r>0$, Theorem 7, Lemma 9, and Theorem 10 of [4] yield $f^{2 r+2}(z)=$ $\left[A\left(\left(z-z_{1}\right)\left(z-\bar{z}_{1}\right)\right)^{r+1}\right]^{r}\left[\left(z-\bar{z}_{1}\right) /\left(z-z_{1}\right)\right]^{n}$ for some integer $n$ and $z_{1} \neq \bar{z}_{1}$. Without loss of generality $z_{1} \in \Pi^{+}$. Thus $r+1$ divides $n$, and $f^{2}(z)=$ $A^{r /(r+1)}\left(\left(z-z_{1}\right)\left(z-\bar{z}_{1}\right)\right)^{r}\left[\left(z-\bar{z}_{1}\right) /\left(z-z_{1}\right)\right]^{m}$ for some integer $m$ and for some $r /(r+1)$ root of $A$. Since $f$ is meromorphic in a neighborhood of $z_{1}$, either $m=r$, or $f$ has a pole or zero at $z_{1}$. If $m=r, f(z)=A^{r / 2(r+1)}\left(z-\bar{z}_{1}\right)^{r}$, rational. If $m \neq r, f^{2}$ has a pole or zero of even order, and so $r-m=2 k$ is even. $r+m=$ $2(k+m)$, and $f(z)=A^{r /(2 r+2)}\left(z-z_{1}\right)^{k}\left(z-\bar{z}_{1}\right)^{k+m}$ which is rational.

If $r<0$, then $1 / f$ is an automorphic form of degree $-r$, and the preceding may be applied to conclude $1 / f$ and thus $f$ is rational.

Example. $M$ finite order elliptic: $g(z)=\exp f(z)$ where $f(z)$ and $M$ are as in Example (c) after Theorem 3.

Theorem 8. Suppose $f(z)$ is an automorpbic form of degree $r$ on $\Gamma$, with multiplier system $v(M)$ and is meromorpbic on $\Omega$ open, where $\bar{\Pi}^{+}-\left\{x_{1}\right\} \subset \Omega$. Then additionally admitted to the conclusion of Theorem 7 are byperbolic matrices sucb that $c=0$, and parabolic matrices.

Proof. Since $\Pi^{+} \subset \Omega, x_{1}$ is real. For infinite order elliptics the above proof remains valid. For hyperbolics with $c \neq 0$, Figure 2 shows there are fundamental regions which lie in $\Omega$. This, as above, is sufficient. 
Examples. (a) $M$ hyperbolic with $c=0: M=\left(\begin{array}{cc}1 / 2 & 1 \\ 0 & 2\end{array}\right), r=1, !(z)=$ $\sum_{l=-\infty}^{\infty} 1 / 2\left(M^{l} z-2 / 3\right) 2^{l} . f(z)$ is meromorphic in $\mathbf{C}-\{2 / 3\}$. (b) Parabolic with $c \neq 0: M=\left(\begin{array}{rr}3 / 2 \\ 1 / 2 & -1 / 2 \\ 1,2\end{array}\right), r$ even $>2$,

$$
f(z)=\sum_{l=-\infty}^{\infty} \frac{M^{l} z-1}{\left(c_{l} z+d_{l}\right)^{r}} \text { where } M^{l}=\left(\begin{array}{ll}
a_{l} & b_{l} \\
c_{l} & d_{l}
\end{array}\right) .
$$

$f(z)$ is meromorphic in $\mathbf{C}-\{1\}$, and a form of degree $-r$.

The two examples are demonstrated in similar manners. Here we consider (a), hyperbolic with $c=0$. For (b) see [3, pp. 72-77]. Let $M=\left(\begin{array}{cc}d^{-1} & b \\ 0 & d\end{array}\right), d>1$, $b \neq 0, x=b d /\left(d^{2}-1\right), 0<a<1, A=\left\{z|\alpha \leq| z-x \mid \leq a^{-1}\right\} . x$ is the finite fixed point of $M$.

In the figure, $c=0, M$ takes any annulus to the next smaller annulus; i.e., toward the center $x$. Thus $\left|\left(M^{l} z-x\right) / M^{l} z\right|$ tends uniformly on $A$ to 1 as $l$ tends to negative infinity, since $\left|M^{l} z\right| \rightarrow \infty$ uniformly in $A$. For all but a finite number of negative $l,\left|\left(M^{l} z-x\right) / M^{l} z\right|<2$ for all $z$ in $A$. Let $\Sigma^{\prime}$ mean those finite number of $l$ are omitted.

$$
\sum_{l=0}^{-\infty}\left|\left(\frac{M^{l} z-x}{M^{l} z}\right) d^{l}\right| \leq \sum_{l=0}^{-\infty} 2 d^{l}
$$

which converges since $d>1$. Thus $\Sigma_{l=0}^{\prime-\infty}\left(\left(M^{l} z-x\right) / M^{l} z\right) d^{l}$ converges uniformly in $A$ to a holomorphic function. Consequently, the undeleted sum $\Sigma_{l=0}^{-\infty}\left(\left(M^{l} z-x\right) / M^{l} z\right) d^{l}$ is meromorphic in $A$.

For the teims with $l$ positive, we use $x=M^{l} x$ to find

$$
\begin{aligned}
\left|\left(M^{l} z-x\right) d^{l}\right| & =\left|\left(M^{l} z-\dot{M}^{l} x\right) d^{l}\right| \\
& =d^{l} \mid d^{-2 l} z+b\left(d^{-1}+d^{-3}+\cdots+d^{-2 l+1}\right) \\
& \quad-\left(d^{-2 l} x+b\left(d^{-1}+d^{-3}+\cdots+d^{-2 l+1}\right)\right) \mid \\
& =d^{-l}|z-x| \leq d^{-l} \alpha^{-1} \quad \text { for } z \text { in } A .
\end{aligned}
$$

Choose $\epsilon>0$ such that $|x|-\epsilon>0$. For all except a finite number of positive $l$, $\left|M^{l} z\right|>|x|-\epsilon$ for all $z$ in $A$, because $M^{l} z \rightarrow x$ uniformly in $A$. Again $\Sigma^{\prime}$ denotes the omission of these finite number of exceptional l. $\sum_{l=1}^{\prime \infty}\left|\left(\left(M^{l} z-x\right) / M^{l} z\right) d^{l}\right| \leq$ $\sum_{l=1}^{\prime \infty} d^{-l} \alpha^{-1} /(|x|-\epsilon)$ which converges since $d>1$. As above, $\sum_{l=1}^{\infty}\left(\left(M^{l} z-x\right) / M^{l} z\right) d^{l}$ is thus meromorphic on $A$.

Define $f(z)=\sum_{l=-\infty}^{\infty}\left(\left(M^{l} z-x\right) / M^{l} z\right) d^{l} . f(z)$ is meromorphic on $A$, and in fact on $C-\{x\}$ since we may let a shrink. It has poles at each point of the infinite set $\left\{z \mid M^{l} z=0\right.$ for some $\left.l\right\}$. Also $f(z)=(O z+d) f(M z)$ so that $f$ is an automorphic form on $\Gamma=\langle M\rangle$.

Theorem 9. Suppose $f(z)$ is an automorphic form of degree $r$ on $\Gamma$, with multiplier system $v(M)$, and is meromorphic on $\Omega$, open, where $\overline{\Pi^{+}}-\left\{x_{1}, x_{2}\right\} \subset \Omega$. 
Then additionally admitted to conclusions of Theorem 8 are byperbolics with $c \neq 0$. That is, the only type ruled out is infinite order elliptics.

Proof. Since $\Pi^{+}$is a subset of $\Omega$, both $x_{1}$ and $x_{2}$ are real. The infinite order elliptics are ruled out as in Theorem 7. Examples have been previously given for all types except for hyperbolics with $c \neq 0$.

Example. Hyperbolic with $c \neq 0: M=1 / 4\left(\begin{array}{rr}5 & -3 \\ -3 & 5\end{array}\right), r$ even $>2, f(z)=$ $\sum_{l=-\infty}^{\infty}\left(M^{l} z-1\right)\left(M^{l} z+1\right) /\left(c z+d_{l}\right)^{r} . f(z)$ is meromorphic in $\mathbf{C}-\{1,-1\}$, and an automorphic form of degree $-r$. For verification, see [3, p. 78].

V. Nonintegral degree. Theorem 6 is generalized.

Theorem 10. Suppose $f(z)$, not identically zero, is an automorphic form of nonintegral degree $r$ with respect to a group $\Gamma \subset \mathrm{SL}(2, R)$, and has multiplier system $v(M)$. Suppose furtber that $f$ is meromorphic in all of $\mathbf{C}$. Then

(i) if $|v(M)|=1 \forall M \in \Gamma$, then $\Gamma$ contains at most translations;

(ii) otherwise either $\Gamma$ contains at most translations, or $\Gamma$ consists solely of byperbolic elements fixing some fixed real $x$ and $\infty$, and $f(z)=k(z-x)^{n}$ for some constant $k$ and integer $n$, and $v(M)=a^{2 n-r}$ where $M=\left(\begin{array}{cc}a & b \\ 0 & 1, a^{\prime}\end{array}\right)$. Thus $b /([1 / a]-a)$ must be the same for every $M \in \Gamma$.

Proof. It is clear that $c=0$, since otherwise examine the analytic continuation once around a small annulus about $-d / c$ of $f(z)=(c z+d)^{r} f(M z)$. The left side returns to its value upon continuation while the right side does not. Thus $c=0$, and $M=\left(\begin{array}{cc}a & b \\ 0 & 1 / a\end{array}\right)$. The case $a= \pm 1$ is case (i) or the first part of case (ii). If $a \neq \pm 1$, then $M$ has a fixed point at $b /((1 / a)-a) . f(z)$ has a Laurent expansion $f(z)=\Sigma_{-l}^{\infty} a_{n}(z-b /((1 / a)-a))^{n}$ valid in some deleted neighborhood of $b /((1 / a)-a) . \quad f(z)=v(M)^{-1}(O z+1 / a)^{r} f(M z)$ becomes

$$
\begin{aligned}
& \sum_{-l}^{\infty} a_{n}\left(z-\frac{b}{(1 / a)-a}\right)^{n}=v(M)^{-1}(1 / a)^{r} \sum_{-l}^{\infty} a_{n}\left(M z-\frac{b}{(1 / a)-a}\right)^{n} \\
& =v(M)^{-1}(1 / a)^{r} \sum_{-l}^{\infty} a_{n}\left(a^{2}+b a-\frac{b}{(1 / a)-a}\right)^{n} \\
& =v(M)^{-1}(1 / a)^{r} \sum_{-l}^{\infty} a_{n}\left(\frac{a^{2} z+b a-a^{4} z-a^{3} b-a b}{1-a^{2}}\right)^{n} \\
& =v(M)^{-1}(1 / a)^{r} \sum_{-l}^{\infty} a_{n}\left[a^{2}\left(\frac{z\left(1-a^{2}\right)}{\left(1-a^{2}\right)}-\frac{a b}{1-a^{2}}\right)\right]^{n} \\
& \quad v(M)^{-1}(1 / a)^{r} \sum_{-l}^{\infty} a_{n} a^{2 n}\left(z-\frac{b}{(1 / a)-a}\right)^{n}
\end{aligned}
$$

Thus $a_{n}=(1 / a)^{r} a^{2 n} a_{n} v(M)^{-1}$ for all $n$. If $f \not \equiv 0$, then $a_{n} \neq 0$ for some $n$, so that 
$(1 / a)^{r} a^{2 n}=v(M)$. But $r$ is nonintegral, and so if $|v(M)|=1$, then $a= \pm 1$. This proves case (i). Suppose now $|v(M)| \neq 1$ for some $M$ and further that there are $n$ and $m$ such that $a_{n} \neq 0$ and $a_{m} \neq 0$. Then $v(M)^{-1}(1 / a)^{r}=a^{-2 n}=a^{-2 m}$. Since $|a| \neq 1, n=m$. Case (ii) follows.

Examples.

(i) $M=\left(\begin{array}{ll}1 & b \\ 0 & 1\end{array}\right), f(z)=e^{i z}, v(M)=e^{i b}, \Gamma=\langle M\rangle$,

(ii) $M=\left(\begin{array}{ll}1 & b \\ 0 & 1\end{array}\right), f(z)=e^{z}, v(M)=e^{b}, \Gamma=\langle M\rangle$,

(ii') $M=\left(\begin{array}{cc}a & b \\ 0 & 1 / a\end{array}\right), f(z)=[z-b /((1 / a)-a)]^{n}, v(M)=a^{2 n-r}, \Gamma=\langle M\rangle$.

\section{BIBLIOGRAPHY}

1. C.W. Curtis and I. Reiner, Representation theory of finite groups and associative algebras, Pure and Appl. Math., vol. 11, Interscience, New York, 1962. MR 26 \#2519.

2. L. Ford, Automorphic functions, Chelsea, New York, 1951.

3. D. James, Automorphic forms on domains larger than the upper half plane, and factors of automorphy, Ph. D. Thesis, University of Wisconsin, Madison, Wis., 1970.

4. M. Knopp, Polynomial automorphic forms and nondiscontinuous groups, Trans. Amer。 Math. Soc。123 (1966), 506-520。 MR 34 \#341.

5. J. Lehner, A short course in automorphic functions, Holt, Rinehart, and Winston, New York, 1966. MR 34 \#1519.

6. H. Poincaré, Mémoire sur les fonctions fuchsiennes, Acta Math. 1 (1882), 193294; Oeuvres. Vol.2, Gauthier-Villars, Paris, pp.169-257.

DEPARTMENT OF MATHEMATICS, WAYNE STATE UNIVERSITY, DETROIT, MICHIGAN 48202 